\title{
DHAKSHA,THE UNMANNED AIRCRAFT SYSTEM IN ITS NEW AVATAR- AUTOMATED AERIAL INSPECTION OF INDIA'S TALLEST TOWER
}

\author{
Dr.K.Senthil Kumar ${ }^{1}$, A.Mohamed Rasheed ${ }^{2}$, R.Krishna Kumar ${ }^{3}$ Mahesh Giridharan $^{4}$ and Ganesh $^{5}$ \\ ${ }^{1}$.Associate Professor ${ }^{2}$.Anna Centenary Ph.D Scholar ${ }^{3}$.Ph.D Scholar ${ }^{4}$.P.G Student 5.P.G Student \\ Division of Avionics,Department of Aerospace Engineering,MIT Campus of Anna University,Tamil Nadu, India
}

ksk_mit@rediffmail.com ksk_mit@annauniv.edu

KEY WORDS: Rotary Wing, Co-axial Quad, UAS, Civil Applications, Aerial Tower Ispection, Low Cost Surveillance

\begin{abstract}
:
DHAKSHA, the unmanned aircraft system (UAS), developed after several years of research by Division of Avionics, Department of Aerospace Engineering, MIT Campus of Anna University has recently proved its capabilities during May 2012 Technology demonstration called UAVforge organised by Defence Research Project Agency, Department of Defence, USA. Team Dhaksha with its most stable design outperformed all the other contestants competing against some of the best engineers from prestigi ous institutions across the globe like Middlesex University from UK, NTU and NUS from Singapore, Tudelft Technical University, Netherlands and other UAV industry participants in the world's toughest UAV challenge. This has opened up an opportunity for Indian UAVs making a presence in the international scenario as well. In furtherance to the above effort at Fort Stewart military base at Georgia,USA, with suitable payloads, the Dhaksha team deployed the UAV in a religious temple festival during November 2012 at Thiruvannamalai District for Tamil Nadu Police to avail the instant aerial imagery services over the crowd of 10 lakhs pilgrims and also about the investigation of the structural strength of the India's tallest structure, the $300 \mathrm{~m}$ RCC tower during January 2013. The developed system consists of a custom-built Rotary Wing model with on-board navigation, guidance and control systems (NGC) and ground control station (GCS), for mission planning, remote access, manual overrides and imagery related computations. The mission is to fulfill the competition requirements by using an UAS capable of providing complete solution for the stated problem. In this work the effort to produce multirotor unmanned aerial systems (UAS) for civilian applications at the MIT, Avionics Laboratory is presented
\end{abstract}

\section{INTRODUCTION}

The unmanned aerial system (UAS) based remote sensing with low-weight imaging systems offers low-cost and flexible tools for the various civilian applications. Team DHAKSHA's current prototype design looks like a eight rotor helicopter, which a single person can carry and operate reliably and safely in adverse weather conditions, providing instant imagery anytime an aerial vantage point is needed. The rotorcraft weighs less than $3 \mathrm{~kg}$, hovers quietly overhead for more than 40 minutes and can patrol at speeds up to $60 \mathrm{kmph}$. The operator uses a small portable control unit to command it to hover or fly in any direction at varying speeds. As crazy as that sounds, the design is incredibly stable and also features ground-tracking as well as obstacle avoidance capabilities. Operator training and workload is minimized through a streamlined design and user interface. Video imagery is transmitted to the handheld ground control station and distributed to decision makers for real-time viewing.

Further, the model can also be scalable into various sizes for tracing holed up militants in various anti terrorism and homeland security applications. The team is also exploring the various possibilities of self powered solar UAVs and UAV for radiation surveillance in and around nuclear power stations and unmanned riot control applications.

As the world is increasingly moving towards automation and from manned to unmanned systems, the system can be deployed for various cartographical, agriculture, forestry, urbanism, traffic management, civil safety, protected areas management, hydrology, geology and environmental applications.

\section{AIM AND SCOPE OF THE WORK}

The following are the goals for Multi-rotor UAV

i. Stable, autonomous hover with active controls system taking dynamic information from sensors mounted on craft.

ii. Stable hover, with addition of variations in altitude based on human input for thrust.

iii. Stable hover, with addition of variations in altitude and directional movement based on human inputs for thrust, pitch, roll, yaw.

iv. Ability to fabricate in-house (Division of Avionics, Department of Aerospace Engineering, MIT Campus)

v. Provide real time stable Video/Images to ground control station

\section{EXPERIMENTAL WORK}

This section discusses the hardware used in the development of the multirotor. Hardware platform construction is the core in building a UAV system. Although the miniature rotorcrafts UAVs are diversified in size, shape, payload, and application purpose, all of them share a similar system configuration. More specifically, a complete mini rotorcraft UAV system consists of the following four parts: 
(i). A radio-control (RC) system

(ii). An avionic system for collecting in-flight data, performing automatic control laws, executing mission-oriented tasks, and communicating with the ground station

(iii) A manual control system consisting of a pilot and a wireless joystick

(iv) A ground station system for monitoring the flight states of the UAV and communicating with the avionic system

The commercially available some hardware components have been used in this research

\subsection{Test Bench}

After all the parts are assembled together the final system looks like the one shown in Figures 1-4

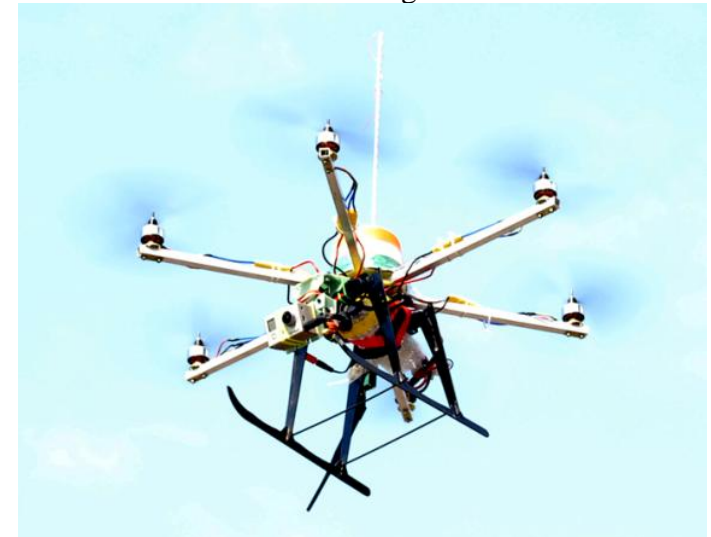

Figure 1. Fabricated Hexrotor in flying mode

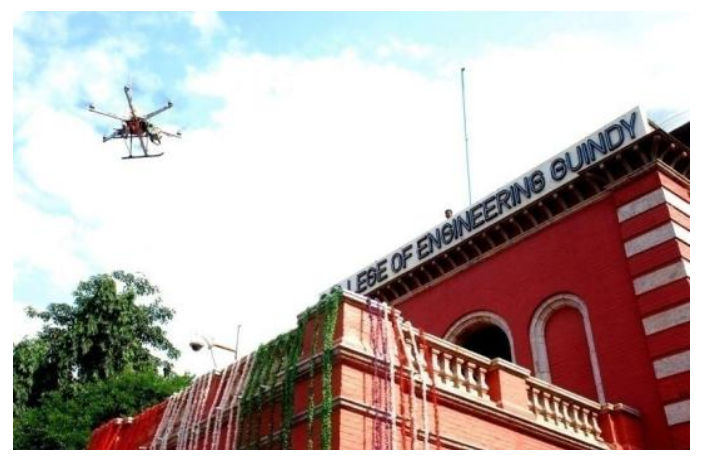

Figure 2

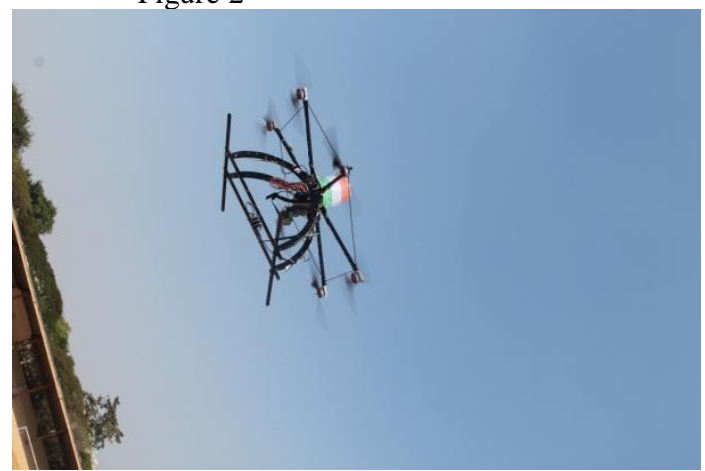

Figure.3:1000 mm Hex rotor

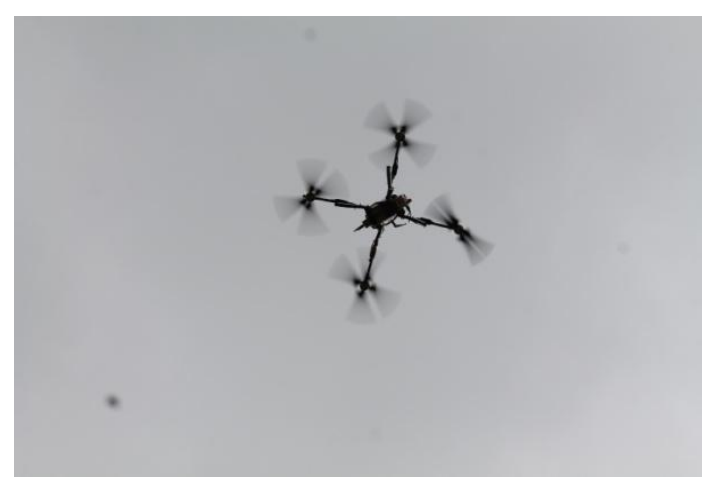

Figure $4.600 \mathrm{~mm}$ carbon rod coaxial quad

After the multi rotor assembly finished the options of experimental setup is considered. One of them is hanging up the quadrotor body with a rope. This keeps the system at a constant height. The two other translations and the three orientations are free. However this setup has a disadvantage. When the system sways sideways the connected rope creates a decoupling action which stabilizes the overall system to certain extend. Another option of experimental setup is the one which has a spherical joint. It prevents the three translations but not three rotations. The available spherical joints tested but their friction rates found to be effectively high. Finally, rope assembly decided to be used in the test bench. Instead the rope passed through an opening in the top PCB gyro board and connected to the center of the cross frame, which reduced the couples that had been caused when an offset was given to the rope-frame connection.

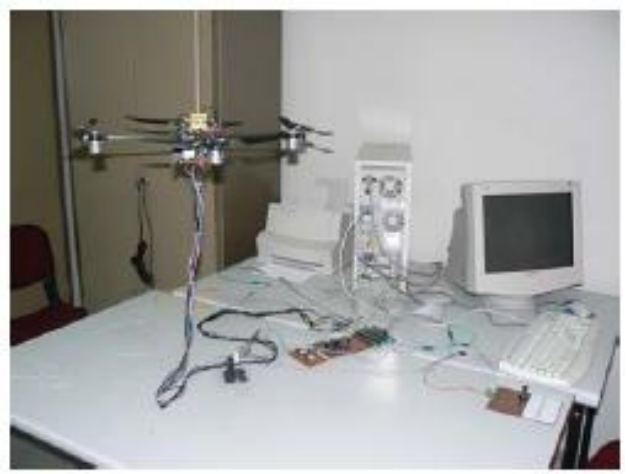

Figure 5. Experimental setup

The final system is hanged with a rope from the center of gravity. And each motor end is loosely supported with ropes in order to prevent large, uncontrolled sways and establish safety during working. The bundle of signal cables that are extended from the quadrotor are winded together and extended close to the axis of center of gravity in order to reduce the torques created by the wires.

\section{TEST RESULTS}

4.1 Eliminating Sensor Noise

Vibration Noise is intrinsically present in the accelerometers. In order to filter that noise 8th order Butterworth filters are placed at the outputs of the accelerometer in the SIMULINK code. Before and after filtering of the accelerometer outputs are given in Fig 6\&7. Note that the disturbance is given by pushing the system on $\mathrm{x}-\mathrm{y}$ plane. 


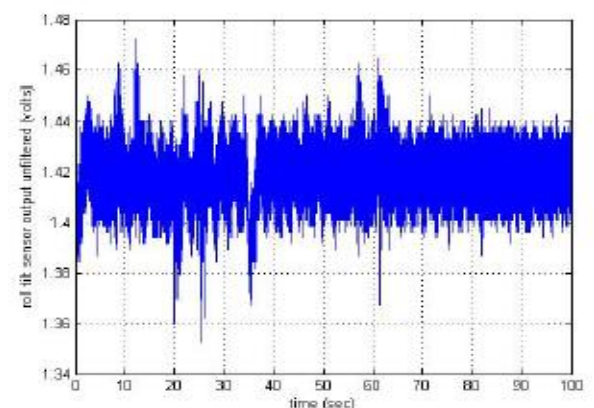

Figure 6 Unfiltered sensor output for roll angle

In Figure 6 it is clearly seen that the signal is almost disappeared under the noise. After the signal filtered with an 8th order Butterworth low pass filter the signal becomes distinguishable and the pattern of motion can be seen (Figure 7)

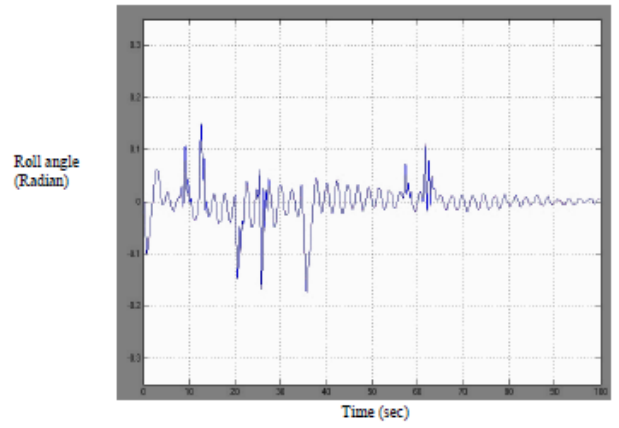

Figure 7. Filtered Roll angle measurement with a 8th order Butterworth low pass filter with $10 \mathrm{~Hz}$ bandwidth

\section{GROUND CONTROL STATION}

The last essential part of the overall unmanned system is the ground control station. Its main responsibility is to realize effective communications between the avionic system and the ground users and pilots. To fulfill this aim, the ground station is generally required to have the following fundamental capabilities: (i) displaying and monitoring the inflight status, (ii) displaying images captured by the onboard system,(iii) generating and updating flight trajectories, (iv) sending control commands to the avionic system, (v) facilitating the ground piloted control or automatic control, especially in unexpected situations such as emergency landing and cruise, and (vi) logging inflight data.

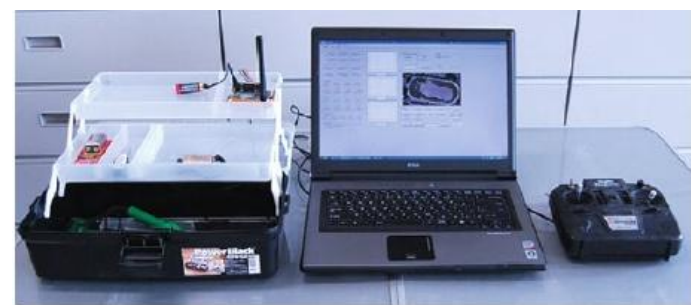

Figure 8. Ground control station set up

\section{NAVIGATION, GUIDANCE AND CONTROL}

The UAV is built with the idea that it can be controlled in both autonomous and radio controlled mode. The team has decided to implement autonomous mode to increase the versatility of the entire system and also for out of sight and long distance missions, (if required). Manual overriding of Autonomous mode to Manual mode and vice versa also programmed.The NGC Block diagram is as shown in Fig.9:

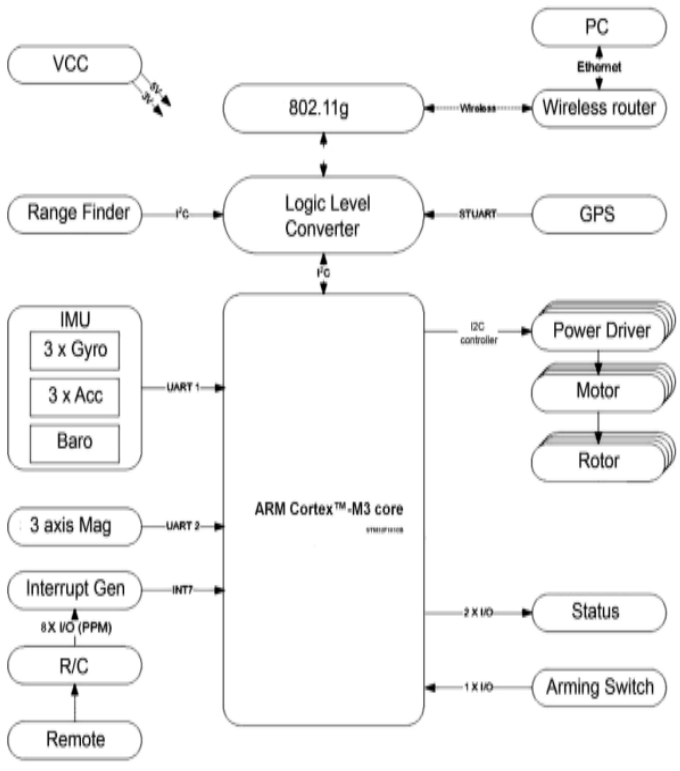

Figure 9. The NGC Block diagram

\subsection{Wirelees Data Transmission}

The camera is tested within our campus and it is interfaced with a video overlay board transmitter and a receiver at the GCS. The GCS receives the real-time video signal from the camera transmitter and using a TV Tuner card, the still images and videos captured and stored.The video being captured now can be used for the purpose of identification of the target and in surveillance operations.

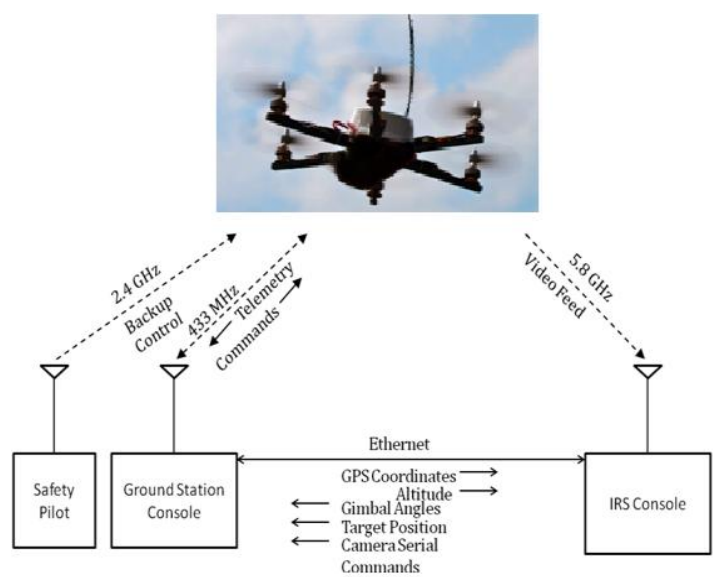

Figure 10. Wireless communication \& Data links

\subsection{Automatic Antenna Tracker}

The Automatic Antenna Tracker is a device which is used to orient the patch antenna as such the orientation of the flight path. It consists of AAT Driver and Teleflylite module. The Teleflylite modules have a microcontroller which is used to decode the NMEA Protocol string from a GPS receiver. Mean 
while it impose GPS meaning full information such as latitude,, Longitude, heading, distance, and number of visible satellites information's are over layer on the audio Channel of the video transmitter using typical modulation. The AAT driver on the ground station will decode the meaning full information from the Audio channel and drive the servo and stepper motor for the exact Azimuth and elevation orientation of the Patch antenna result in the Antenna will always faces the orientation of the flight heading path.

\section{CASE STUDY: AERIAL INSPECTION OF INDIA'S TALLEST RCC TOWER}

The presentation is about the investigation of the structural strength of the India's tallest structure, the $300 \mathrm{~m}$ RCC tower using an UAV. Unmanned inspection devices are a new way to inspect tall structures, thereby increasing the number of daily inspections while eliminating the risk of personal injury. The system provides the aerial platform for any type of viewing and recording, for determining present conditions, and/or for documentation of completed repairs and inspections, etc

The ability to survey the area from above provides an "eye in the sky" perspective on current conditions or damages. Features of the vehicle include full auto take-off, waypoint (GPS) tracking, and auto landing. The ability to know the exact location of the camera at any given moment allows for complete documentation while the inspection is in progress.
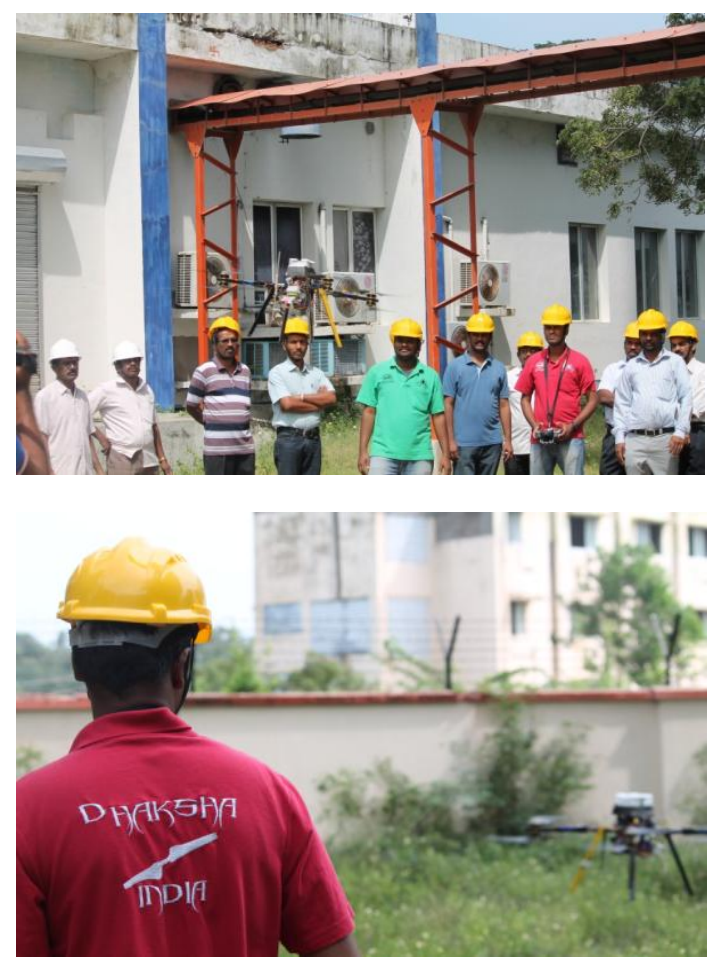

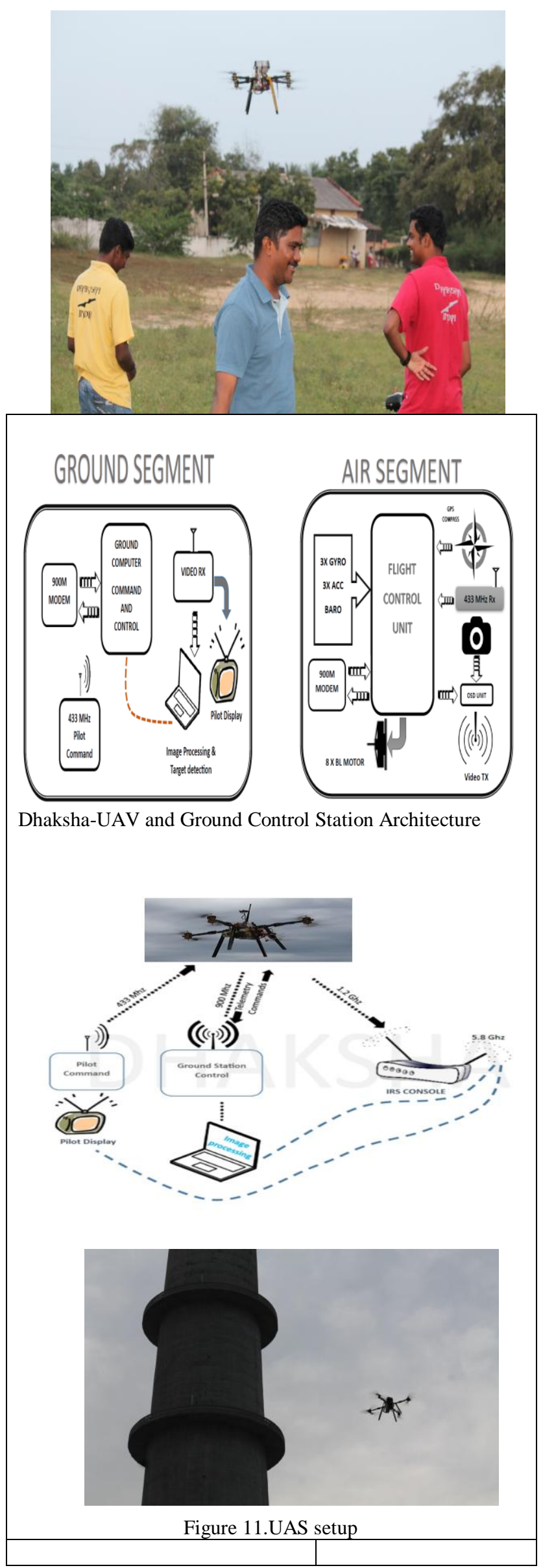




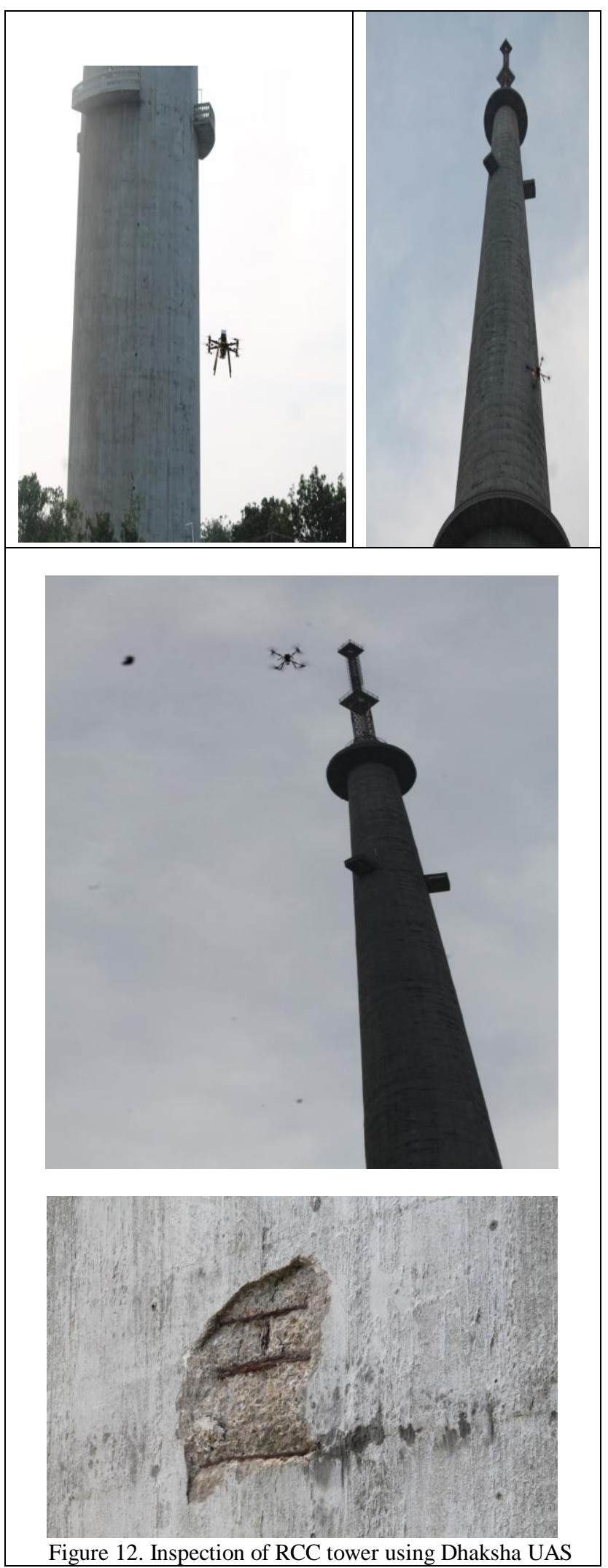

The UAS architecture is mainly divided into two Segments namely Aerial Segment and Ground Segment. The aerial segment consist of UAV with surveillance/monitoring payloads which do the complete surveillance and can transmit in those sensor/video feed to ground station for control, Navigation, to guide the UAV also for viewing and post data processing of captured video/image.

The responsibility of ground control station is to realize effective communications between the avionic system and the ground users/pilots.

Using the ground control software, we are providing circular pattern waypoint which the UAV must fly through. In GCS, we have the provision to simulate the UAV mission before actually flying the real UAV.
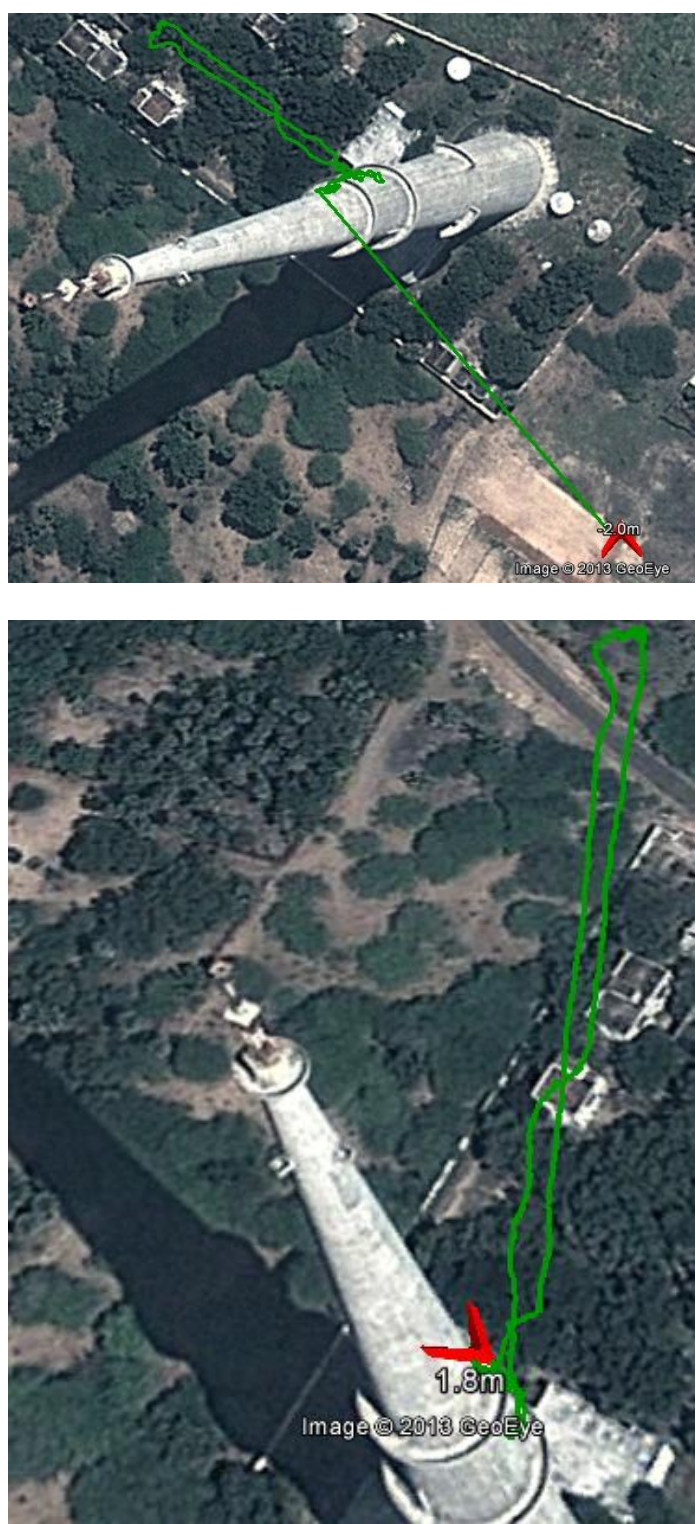

Figure 13. Tower testing:UAV Mission Plan

The primary objective of the mission was to capture clear and detailed images/videos of the tower structure for efficient crack mapping of entire outer surface of the tower.On deployment, within five minutes of take off Dhaksha reached the top of the 300 meters tall tower, carefully examining the structure from many angles. When performing the tower inspection, the first step was to achieve a position adjacent to the components of interest. This can be quickly achieved by positioning the UAV 
as close to the tower and below the desired final position, and taking off to climb to the desired height.

The system flew around the tower in circular and vertical flight for closer and wider angle views. As it flew, it filmed in HD video. The vehicle was able to see the flaws in the structure viz., cracks, internal structural damage, steel frame exposure and rusting due to sea breeze erosion, peeling off and caving in, etc., The vehicle automatically maintained a position hold while the operator pointed the camera at each point of interest. Clicking on the component of interest in the video stream sent a high resolution image/video to the GCS for immediate and post analysis. The ground control station received live video/images and is able to see what the UAV sees as it flies. Skilled technicians monitored the flight and requested the UAV pilot to go back or to zoom in as it flies. Because the GPS location and altitude are visible on the ground control station as it flies, you can pinpoint the areas needing attention, repair, or just another look.

Critically, in contrast to the traditional joystick operated small UAVs the DHAKSHA's map based click and go navigation system allowed the operator to focus on the inspection rather that the flying. While this is being done all personnel were safely on the ground, incurring no risk whatsoever, the main objective of an aerial unmanned mission. The cost of inspection is reduced and the quality of the inspections has actually been improved because the UAV uses better optics to view and record what it sees.

\section{CONCLUSION}

At the Institute, a family of autonomous Multi rotor Aerial Vehicles was developed. The aim of the project was to have a fully functional multi-rotor helicopter capable of autonomous operations. During the test runs we achieved lift of the craft, and some level of autonomous hover, with visible corrections from the control system. However, noise from the tilt sensors provided enough uncertainty that we were not willing to attempt flight without a cable to secure the craft from flipping over and losing altitude.

We feel that our design and construction of the physical structure was a success both in terms of stability and strength of the craft, and in terms of weight reduction.. We also succeeded in writing fully functional software for the control of the motors. We implemented this software code on a commercially available microcomputer and obtained visible results with an oscilloscope set up to read the sensor and operator inputs and PWM outputs.

The UAV was controlled by setting waypoints on a digital map by means of special software running on a common laptop or Tablet PC. Telemetry link from Ground Control to the aircraft is currently done with a dedicated radio modem with short range $(5000 \mathrm{~m})$, but due to the modular design, replacement by other telemetry hardware with much higher range is comparably easy. Payload data can be stored on solid state memory cards, allowing high volumes of data storage and easy handling of data after landing.

Three prototypes are available for civil applications it has a payload capacity of 200-1500 grams and is suitable for many purposes.. Finally being part of a team that establishes a working test bed for the Anna University, MIT, Avionics Laboratory has been a great experience.

The scope of growth in the Civilian applications could be even larger as has been seen in the past eg: Internet, Civil aviation,
IT which were initially military applications but are a part and parcel of the common man's life today. Having developed prototypes of varied dimensions indigenously and given our proven track record, the ability to meet any of the customer mission requirements any time at short notice and reasonable costs would be our major strength.

\section{REFERENCES}

[1] S.Sassen, P. Uhleman, Quattrocopter A unique MicroAerial Vehicle, European Aeronautic Defense and Space Company Corporate research centre, November 2008.

[2] P.Pounds , R.Mahony, J.Gresham, Towards DynamicallyFavourable Quad- Rotor Aerial Robots, Australian National University, Canberra, Australia, 2009.

[3]Nagai, M., Chen, T., Shibasaki, R., Kumgai, H., Ahmed, A., 2009. UAV-borne 3-D mapping system by multisensory integration. IEEE Trans. Geosci. Remote Sens. 2009 (47): 701-708.

[4] Aber, J. S., Marzloff, I., Ries, J. B., 2010: Small-Format Aerial Photography. Principles, Techniques and Gesoscience Applications. Elsevier, Amsterdam.

[5] Honkavara, E., Hakala, T., Saari, H., Markelin, L., Mäkynen, J., Rosnell, T., 2012b. A process for radiometric correction of UAV image blocks. Photogrammetrie, Fernerkundung, Geoinformation (PFG) 2/2012, pp. 115-127.

\section{ACKNOWLEDGEMENT}

The authors are grateful to Dr.Kaliraj, Vice Chancellor, Anna University and Dr.Thamarai Selvi, Dean, MIT for providing organizational facilities for carrying out this research work at Madras Institute of Technology Campus of Anna University. We would like to express our sincere thanks \& gratitude to Prof.Dr.Umarani and their Structural Engineering team,Department of Civil Engineering,Anna University for providing the experiment field and general cooperation for this research work. We also express special thanks to Dr.Sekar,Dean,CEG and Prof.Dr.Samual Knight for their technical suggestions on aerial inspection.We would like to convey our thanks to Dr.B.T.N Sridhar,Head,Dept. of Aerospace Engg.MIT for his inspiration and encouragement throughout the project and wishes to all our Avionics students.

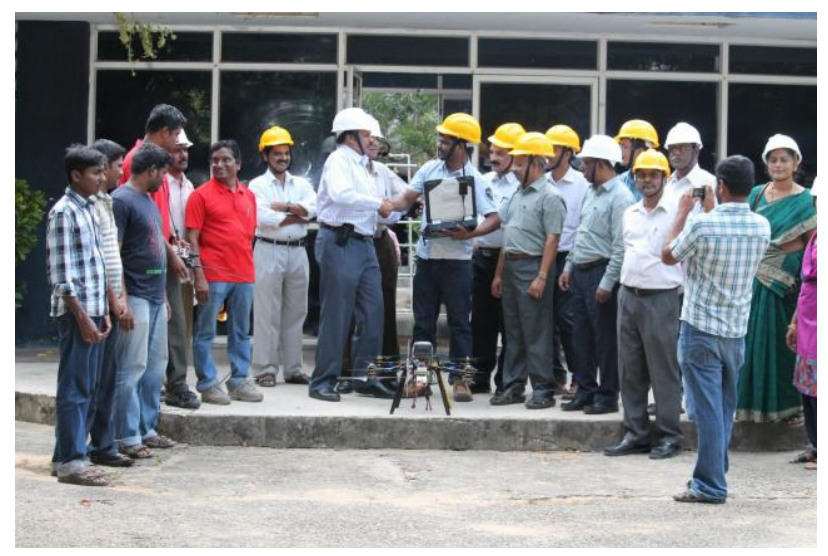

